\title{
Response: Randomised trial of the effect of a gastrin/CCK2 receptor antagonist on esomeprazole-induced hypergastrinaemia: evidence against rebound hyperacidity
}

\author{
Malcolm Boyce $^{1}$ (D) Frans van den Berg $^{1} \cdot$ Toni Mitchell $^{1} \cdot$ Kate Darwin $^{1}$. \\ Steve Warrington ${ }^{1}$
}

Received: 18 March 2017 / Accepted: 20 March 2017 /Published online: 1 April 2017

(C) Springer-Verlag Berlin Heidelberg 2017

\section{Dear Editor}

We thank Dr. Reeve and Professor Hilmer for their interest in our article [1]. Their comments on 'deprescribing' and 'tapering' of proton pump inhibitor (PPI) treatment are valid and helpful but are not directly relevant to our findings or conclusions.

Tapering the dose of a PPI rather than stopping it abruptly is based on the concept that abrupt withdrawal leads to rebound hyperacidity and dyspepsia secondary to persistent PPI-induced hypergastrinaemia, and that tapering minimises withdrawal symptoms [2]. Although we demonstrated neither rebound hyperacidity [3] nor persistent hypergastrinaemia [1] after abrupt cessation of PPI treatment in healthy subjects, we did not suggest that those negative findings should prompt any change in the clinical management of patients receiving a PPI. Rather, we stated that PPI withdrawal should be studied further in an adequately powered trial in patients on long-term PPI treatment.

We suggest that a multicentre, randomised, double-blind, double-dummy study of a gastrin/CCK2 receptor antagonist in four groups of patients on a PPI for at least 1 year, and ideally at high dose, is required to confirm the existence of rebound hyperacidity/dyspepsia after PPI withdrawal, assess its importance, and whether it is gastrin driven. The treatment groups should be (a) PPI withdrawal plus placebo CCK2RA and placebo PPI; (b) PPI withdrawal plus active CCK2RA and placebo PPI; (c) continuing PPI plus placebo CCK2RA; and (d) continuing PPI plus active CCK2RA [4]. Patients should be stratified for the original diagnosis, PPI dose and type, and serum gastrin concentration, and there should be a run-in period. Outcome measures would be symptoms and serum gastrin and chromogranin A. Measurement of gastric acid secretion would be ideal but would make the study more difficult to do.

Meanwhile, tapering the dose rather than stopping the PPI abruptly may well gain the support of patients on long-term therapy and help reduce over-usage, but robust evidence to justify the need for the procedure is lacking.

\section{References}

1. Boyce M, van den Berg F, Mitchell T, Darwin K, Warrington S (2017) Randomised trial of the effect of a gastrin/CCK2 receptor antagonist on esomeprazole-induced hypergastrinaemia in healthy subjects: evidence against rebound hyperacidity. Eur J Clin Pharmacol 73:129-139

2. Björnsson E, Abrahamsson H, Simrén M, Mattsson N, Jensen C, Agerforz P, Kilander A (2006) Discontinuation of proton pump inhibitors in patients on long-term therapy: a double-blind, placebocontrolled trial. Aliment Pharmacol Ther 24:945-954

3. Boyce M, Dowen S, Turnbull G, van den Berg F, Zhao C-M, Chen D, Black JW (2015) Effect of netazepide, a gastrin/CCK2 receptor antagonist, on gastric acid secretion and rabeprazole-induced hypergastrinaemia in healthy subjects. Br J Clin Pharmacol 79: 744-755

4. Boyce M, Lloyd K, Pritchard DM (2016) Potential clinical indications for a gastrin/CCK2 receptor antagonist. Curr Opin Pharmacol 31:68-75

Malcolm Boyce

mboyce@hmrlondon.com

1 Hammersmith Medicines Research, Cumberland Avenue,

London NW10 7EW, UK 